\title{
Routine follow-up visits not necessary after third molar removal under sedation
}

\author{
Is it necessary for a routine follow-up visit following third molar removal under intravenous \\ sedation?
}

\author{
Sittitavornwong S, Waite PD, Holmes JD, Klapow JC. The \\ necessity of routine clinic follow-up visits after third molar \\ removal. J Oral Maxillofac Surg 2005; 63:1278-1282
}

Design This was a randomised controlled trial (RCT) conducted in the US.

Intervention People were recruited to the trial who required surgical removal of at least two impacted third molars under intravenous sedation on an outpatient basis. They were treated by board-certified oral and maxillofacial surgeons. Participants were divided randomly into two groups: the first was given a 2-week postoperative follow-up appointment, and the other had no follow-up. All patients received postoperative instructions and were contacted by telephone on the day after surgery. At 2 weeks postoperatively, all patients either returned to the clinic or were interviewed by telephone.

Outcome measure Patients were asked to answer a questionnaire on postoperative day 1 and at 2 weeks after their treatment. The questionnaire was originally proposed by Worrall ${ }^{1}$ but was modified for use in this study.

Results Of the 60 consecutive patients who underwent third molar removal, only 48 were included. The mean age was 20 years (range, 15-33 years). There were no significant differences in the number of patients and gender between the groups receiving clinic or telephone follow-up. Seventy-three per cent (35 out of 48 ) out of all the patients preferred telephone follow-up, and 27\% (13 out of 48) of patients preferred clinic follow-up. Eighty-five per cent (29 out of 34) of patients who did not have any complaints on postoperative day 1 preferred telephone follow-up $(P<0.01)$.

Conclusions A routine follow-up visit following third molar removal under intravenous sedation is not necessary in patients aged between 15 and 35 years. Pre-operative and postoperative instructions should be clear, however. A selective review policy may be appropriate if a patient is mentally retarded, is taking psychoactive drugs, or has an intraoperative complications or a has made a complaint via telephone.
Address for correspondence: Dr Sittitavornwong, Department of Oral and Maxillofacial Surgery, University of Alabama at Birmingham, 456 School of Dentistry Building, 1919 7th Avenue South, Birmingham AL 35294, USA. E-mail: sjade@uab.edu

\section{Commentary}

Third molar surgery is one of the most commonly performed dentoalveolar operations in the UK. The decision to review patients undergoing surgery is made by the surgeon and there are few guidelines on this topic: review strategies therefore vary considerably. This RCT examined the necessity of routine follow-up visits. Forty-eight people who underwent surgical removal of third molars under intravenous sedation received a telephone review $24 \mathrm{~h}$ postoperatively and then either telephone or clinic review at 2 weeks after treatment. A questionnaire was completed by the patients at both reviews.

Via the questionnaire, the authors examined the level of patient satisfaction with their reviews, post-operative morbidity and patients' need to seek postoperative help from a clinician. In the results, the authors attempted to determine whether or not a patient's preference for clinic review or telephone review was related to their incidence of postoperative complaints and their presence in the clinic or telephone review group. They found that $73 \%$ of the total number of patients preferred a telephone review. The number of patient complaints was also related to the presence of postoperative complications and the necessity for active treatment.

The aims and objectives of the study are a little unclear, compounded by the absence of the questionnaire from the article. The multiple variables and numerous results, with few tables or graphs, made the results confusing. The authors conclude that a policy of selective review after surgical removal of third molars may be appropriate; they outline a review protocol based upon a 1997 study by the British Association of Oral and Maxillofacial Surgeons. ${ }^{2}$ The review protocol is useful, but there is little mention of the impact an immunocompromised medical status may have upon postoperative healing, and therefore the potential benefit of review in such patients. The authors stress the need for clear, written postoperative instructions in a variety of languages.

The principle of reviewing patients indirectly over the phone may reduce costs in the UK National Health Service by reducing outpatient follow-up appointments, and the widespread use of mobile telephones simplifies verbal contact. Although telephone review may be acceptable to patients, however, it does not allow the surgeon to perform a clinical examination which may reveal postoperative pathology unnoticed by the patient. Regular audit is essential to determine the incidence of postoperative morbidity.

\section{Gillian Ainsworth}

Department of Oral Surgery, Glasgow Dental Hospital and School NHS Trust, Glasgow, Scotland, UK

1. Worrall SF. Are postoperative review appointments necessary following uncomplicated minor oral surgery? Br J Oral Maxillofac Surg 1996;34:495.

2. Pratt CA, Hekmat M, Pratt SD, Zaki GA, Barnard JDW. Controversies in third molar surgery - the national view on review strategies. Br j Oral Maxillofac Surg 1997; 35:319-322.

Evidence-Based Dentistry (2006) 7, 81-82. doi:10.1038/sj.ebd.6400442 